\title{
HEIMDALL: a technological solution for floods and multi-hazard management support
}

\author{
Stéphanie Battiston ${ }^{1 a}$, Monika Friedemann ${ }^{2}$, Benjamin Barth², Jordi Vendrell ${ }^{3}$, David Martin ${ }^{3}$, Sandro Martinis ${ }^{2}$, Flavio \\ Pignone $^{4}$, Christian Knopp ${ }^{2}$, Eva Trasforini ${ }^{4}$, Daniel Milla Gascón ${ }^{5}$, Lorenzo Massucchielli ${ }^{6}$, Nedim Jasic ${ }^{6}$, Julien Briant ${ }^{1}$ \\ and Torsten Riedlinger ${ }^{2}$ \\ ${ }^{1}$ Strasbourg University - ICube SERTIT, 300 boulevard Sébastien Brant, 67412 IIIkirch Graffenstaden, France \\ ${ }^{2}$ German Aerospace Center, Muenchener Strasse 20, 82234 Wessling, Germany \\ ${ }^{3}$ Pau Costa Foundation, Av. Mossèn Cinto Verdaguer, 42 esc. A bxs 2A, 08552 Taradell, Barcelona, Spain \\ ${ }^{4}$ CIMA Research Foundation, Armando Magliotto, 2, 17100 Savona, Italy \\ ${ }^{5}$ Generalitat de Catalunya, Directorate for Police Coordination, Carrer de la Diputació, 355, 08009 Barcelona, Spain \\ ${ }^{6}$ Italian Red Cross, Toscana, 12, 00187 Rome, Italy
}

\begin{abstract}
The Horizon 2020-funded project HEIMDALL (Multi-Hazard Cooperative Management Tool for Data Exchange, Response Planning and Scenario Building) aims to improve immediate and long-term collaborative strategic planning on a regional, national and international scale among affected disaster risk management and response stakeholders. Today, the collective interoperability is not sufficiently achieved during disaster events, which reduces the ability to effectively undertake planning and response actions that require collaborative working among all the stakeholders involved. To address this shortcoming, the project develops a platform that offers a wide range of tools, products and services to support decision-making processes, and in particular different activities in the response planning process for complex multi-hazard crisis situations. The platform is designed in a modular approach, using various data sources as inputs and proposing a set of services that assist command and control centres, first responders and local populations in taking risk-informed decisions during the preparedness and response phases of disaster management. Some of the core functionalities are built around the ability to integrate space-based, ground-based and aerial-based data in order to forecast and monitor floods and other disaster events, and to feed the system with operational and external data sources to provide major inputs to emergency coordination.
\end{abstract}

\section{Context of the HEIMDALL Solution}

Managing floods and other natural and man-made disasters usually involves multiple emergency management organisations, multiple jurisdictions and even multiple countries in case of cross-border events. Following the initial disaster event, cascading effects can further amplify the degree and complexity of disaster situations. This imposes a high need and degree of crossorganisational communication and cooperation, not only during response but also in the preparedness phase, between all stakeholders (civil protection, medical services, police and fire fighting units) working at command and control centres or on the field. However, relevant studies have revealed that collective interoperability is not sufficiently achieved, which reduces the ability to perform collaborative activities, including decision making and action implementation (House et al., 2014). Moreover, information overload and

${ }^{a}$ Corresponding author: s.battiston@unistra.fr

DOI 10.3311/FLOODRisk2020.18.4 uncertainty in a crisis situation hinder situation awareness and decision-making capabilities.

Managing disasters begins before the disaster happens by being ready and aware of hazards, considering risks, possibilities and preventive measures, building scenarios and training. There is clearly a need to ensure efficient societal preparedness, to improve incident and emergency situation response mechanisms in order to minimise the impact on people, property, the environment, and the society as a whole. An element of this improvement should be the provision of a flexible platform for multihazard emergency planning and management to disaster risk management first responders. Such a platform makes use of innovative technologies for the definition of realistic multi-disciplinary scenarios and response plans. Developing such a tool is the ambition of the European Union's Horizon 2020 project HEIMDALL (HEIMDALL, 2017). 
In the following sections the concept of the HEIMDALL project is outlined and demonstrated within the context of flood management.

\section{A Multi-Hazard Cooperative Management Platform}

HEIMDALL, standing for Multi-Hazard Cooperative Management Tool for Data Exchange, Response Planning and Scenario Building, aims to improve immediate and long-term collaborative strategic planning on a regional, national and international scale among the many affected disaster risk management and response stakeholders. The objective is to design and provide decision makers and other stakeholders a platform offering a wide range of tools which facilitate emergency management. In particular, HEIMDALL aims to support technically different typical response planning activities involving complex multi-hazard scenarios (Friedemann et al., 2018). The main solutions that have been designed and implemented include the: (a) support in the creation, analysis and exchange of realistic multi-disciplinary disaster scenarios, (b) provision of more, better, clearer and validated data, (c) recording of conditions, actual events and actions as the situation evolves, (d) analysis of possible futures of a situation and potential consequences to assess the effectiveness of potential working strategies and identify options and contingencies, (e) evaluation and revision of response plans based on lessons learnt from disasters, (f) inter- and cross-organizational communication and sharing of existing knowledge, situational information, disaster scenarios, strategies and response plans including communication to the public. Domain standards are respected where applicable.

HEIMDALL results are the fruit of a wide variety of technological specialists and potential end-users collaborating. End-users from medical emergency services, police, firefighting units, civil protection, command and control centres from different organisations and countries are participating in the project. This enables the platform to address as best as possible the requirements of the different actors involved in disaster risk management. The project addresses some of the main disaster types regularly affecting European countries - floods, forest fires and landslides - including scenarios of cross-border incidents, multi-disciplinary events, interorganisational cooperation and population awareness.

\section{HEIMDALL Services}

The system is designed as an integrated service platform using various data sources as inputs and proposing a set of services that can be useful to control centres, first responders and local populations during the preparedness and response phases.

In order to identify potential products and services, the process how end-users make their decisions was analysed and the outcomes of the different steps were identified and combined. The results are illustrated in Figure 1. The cornered boxes show the steps of the Joint Decision Model (JDM) (Cohen-Hatton et al., 2015) followed by rectangular boxes with the outcome of the respective steps. For instance, the outcome of the "gather information \& intelligence" step is an identified situation or a simulation result whereas the step "identify options and contingencies" results in a plan or a decision. The three phases of the normative models situation assessment (SA), plan formulation (PF) and plan execution (PE) are shown in the background of the modified JDM model. The SA phase corresponds to the gathering of information step and includes the assessment of risk. The development of the working strategy is an interaction of the SA and PF, while the PF persists until a decision is taken. PF is followed by the PE which corresponds taking an action and coordinating it, i.e. executing the decision. To close the cycle, information on the outcome of the action is gathered and consequently the SA starts again. In each of these steps, HEIMDALL functionalities can support decision makers prior, during, and in the aftermath of disaster events. These functionalities are presented in the outer circle indicating with the size of the arcs the supported steps.

Figure 1. HEIMDALL service concept. Source: HEIMDALL deliverable D2.11 (2018).

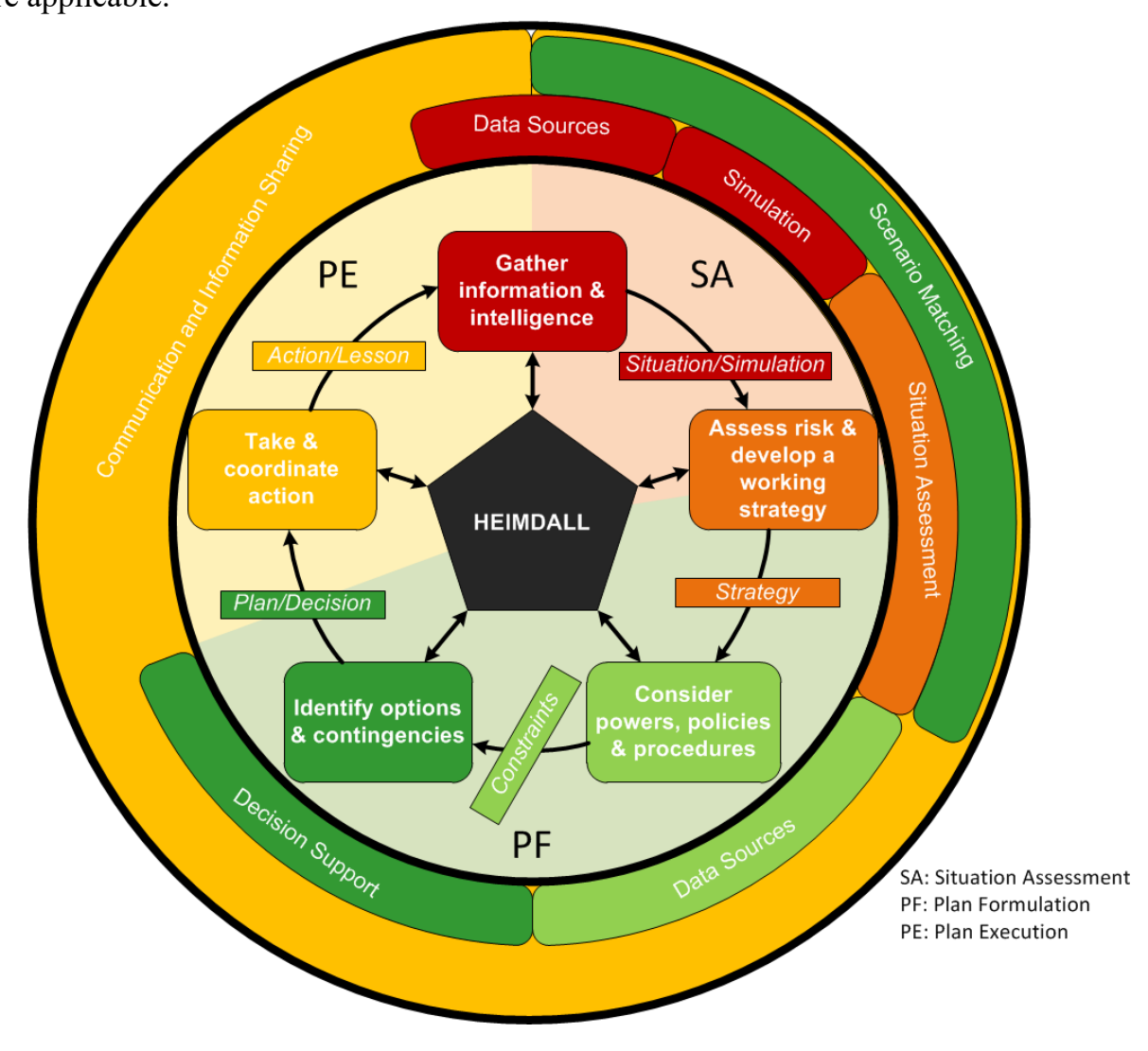


The system enabling this is shown in Figure 2. The approach used in HEIMDALL entails a general shift in environmental systems and in disaster management. In particular, as it strays from isolated monolithic implementations so as to adopt functionally distributed information provision and processing system approaches.

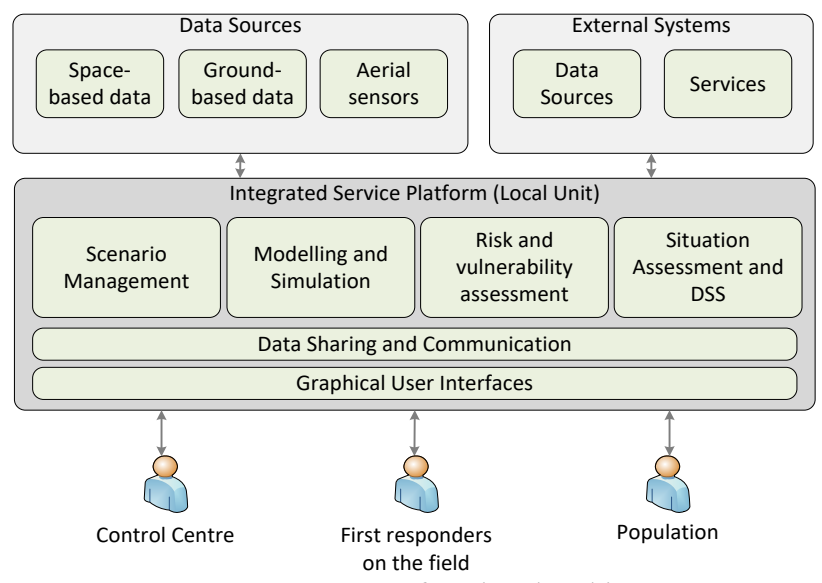

Figure 2. HEIMDALL functional architecture.

Source: HEIMDALL deliverable D2.12 (2018).

Over the last decade, web services have become a major technology for implementing a highly modular architecture. There is an established body of web service interface standard available from the International Organization for Standardization (ISO), the Open Geospatial Consortium (OGC), the World Wide Web Consortium (W3C) and Internet Engineering Task Force (IETF) for interoperable data access and exchange. Visualization, vector and raster data access, along with the ability to search for spatial data is mostly covered by the standards provided by OGC such as the Web Mapping Service (WMS), the Web Feature Service (WFS), the Web Coverage Service (WCS), and the Catalog Service for the Web (CSW).

Encapsulating all HEIMDALL modules in individual web services is the prerequisite for multidisciplinary response and response planning which usually involves various groups of stakeholders in need of different combinations of services. Combining different HEIMDALL services allows for building on top of one another and offering additional features for disaster response and preparedness. The main benefit provided by the system is that it can be easily extended. If a given user wants to access new functionalities at a later moment, they can be integrated as external systems that are already in use.

The platform integrates space-based, ground-based and aerial-based data in order to forecast and monitor disaster events. In the case of floods, products highlighting the flood extent over wide areas are provided thanks to multi-sensor satellite data. Furthermore, the system combines internal data sources like an app for field responders' data with external data sources and services like weather and EFFIS data that can also be inputs.

Scenario management is one of the services offered by the system; it aims at supporting decision makers in building, storing, reviewing and comparing realistic and multidisciplinary scenarios and in tracking decisions, measures taken and lessons learnt. The scenario matching service allows users to find scenarios with a similar context, environmental conditions and hazard behaviour, from local storage as well as shared by other organizations. Scenario matching results allow for exploring similar situations and the linked response plans and lessons learnt.

Modelling and simulation are dedicated to forecasting hazard evolution based on the existing input data. At the moment it is possible to simulate the evolution of flood, forest fire and landslide hazards, but this can be extended to other natural and human-made hazards in the future.

The risk and vulnerability service supports the platform with specific products based on Earth Observation (EO) data and simulated models, assessing the consequences of a situation in terms of both physical and human exposure and impacts.

Situation assessment functionality integrates all relevant information sources to provide a sound and clear composite picture of the hazard impact on people and assets as the situation evolves, including a standardsbased situation summary for overview and sharing.

Decision support generates the information base for decisions related to response-oriented tasks such as contingency management. These functionalities combined provide the possibility for the end-users to perform an analysis of different possible scenarios, e.g. worst-case assumptions.

Communication mechanisms and technologies support information sharing among the relevant stakeholders, including first responders deployed on the field and the population at risk. Satellite-based connectivity in remote areas is provided where terrestrial alternatives are unavailable enabling communication between first responders in the field and back and forth with the control centre. Information can also be shared with other authorities, neighbouring municipalities and countries. Emergency management message standards such as the EDXL (Emergency Data eXchange Language) group of standards will be utilized in order to overcome problems of interoperability and semantic heterogeneity and to ensure the optimal provision of disaster-related information for fast decision-making in a highly coordinated manner (Friedemann et al., 2018).

\section{HEIMDALL and Flood Management}

This section demonstrates how the HEIMDALL services can support risk and emergency management organisations specifically in case of flood events.

\subsection{Scenario management}

Most emergencies follow a generally known pattern and are composed of elements that have some basis in past disasters. Therefore, response plans should be firmly based on scenarios of what is likely to happen (Alexander, 2009). The creation of scenarios can help decision makers to explore the consequences of impacts 
and response actions. The HEIMDALL scenario data model has been co-designed in multiple iterations by the technical and the end-user partners. It provides harmonized attributes and value lists common to all involved groups of end users under consideration of existing terminologies. For instance terms and concepts specified by the EDXL standards (Friedemann et al., 2018) and the EC Floods Directive on flood risk assessment and management (2007/60/EC) that mandates that the description of relevant past flood events has to be recorded using a standardized structure (European Commission - DG Environment, 2013) have been collated and merged. The HEIMDALL scenario data model allows for the specification of different aspects of a disaster situation: a) environmental conditions such as weather concerns, b) hazard characteristics, c) flood (or forest fire/landslide) observations, simulations and impact assessments d) the organizational context and e) strategic plans and decisions. With regards to $b$ ) the data model allows for hazard-specific attribution. For instance, flood events can be classified by the sub-hazard types

- Fluvial Flooding,

- Flash Flooding,

- Storm Surge,

- Estuarine/Coastal,

- Cloudburst,

and described by hazard attributes such as water velocity and water height. As to e) through using the common data structures the user is able to define flood-specific strategies and objectives. Furthermore, the scenario data model includes a harmonized lessons learnt data structure which allows stakeholders to capture experience of the emergency management in complex disasters.

Scenario management helps to improve immediate and long-term cooperative situation assessment and response planning activities (Friedemann et al., 2018). Throughout the decision-making process described in section 3 scenarios act as a mean for preparing for, recording, sharing, evaluating of and learning from disaster situations. The latter is supported by the scenario matching service which allows decision makers to search for similar disaster situations, both, stored in the local HEIMDALL unit as well as shared by other units. This is possible due to the harmonized scenario data structure which provides the same core set of parameters for different hazard types, organizational levels, countries and regions, complexities, scales, etc.

The scenario matching service performs a ranking of scenarios based on the mutual similarity. Distance measures are applied to individual scenario parameters used for matching, the so-called matching criteria. Suitable matching criteria have been identified and formalized by the end user partners and practitioners in the Advisory Board. Matching criteria include:

- Weather conditions and synoptic situation,

- Flood location (i.e. spatial matching),

- Hazard type,
- Scenario status and urgency (e.g. actual vs. fictional, immediate vs. historic),

- Stressed capabilities (e.g. incident command organization, information management, community involvement, etc.),

- Challenge,

- Hazard behaviour.

For every criterion a mismatch is computed which represents the distance of a given value to a scenario parameter value in such a way, that the scenario parameter value corresponds exactly to the compared situation when the distance is zero. For example, the spatial mismatch is calculated by applying the geodesic distance computation to the centroids of the flood location geometries (Karney, 2013). The mismatch of stressed capabilities in the compared situations is computed using the Jaccard similarity (Han et al., 2006). The metric measures the similarity between finite sample sets.

The individual mismatches are combined using the Simple Additive Weighting approach (Behrens et al., 2010; de Brito and Evers, 2016; Goodridge, 2016). The matching can be influenced by adding weights to each criterion. Matching criteria and metrics needs to be (re)evaluated in consecutive tests with the end users while the scenario database gets more and more populated with historic and new scenarios. Therefore, the combination of recording and matching scenarios including lessons learnt from prior incidents can improve the ability of stakeholders to learn and evolve from complex situations and thereby allow them to respond more effectively and operate more efficiently during disasters.

\subsection{Flood simulation and modelling}

The Civil Protection actions in terms of prevention and preparedness (including Early Warning activities) are crucial when dealing with real-time flood risk management.

Due to the fact that several areas, all over the world, are prone to flood risks with consequent effects of millions of euros of damages and human losses generation (UNISDR, 2015, "The human cost of weather related disaster"), in the last decades were more and more developed systems that bring to hydro-meteorological chain (Gabellani et al., 2005; Hally et al., 2015; Molini et al., 2009; Poletti et al., 2017; Siccardi et al., 2005; Silvestro et al., 2011).

The hydro-meteorological forecasting chains operationally used in many countries are very important to know the general behaviour of an event but is not enough to reach the spatial and temporal scale necessary to locally adopt significant measures to anticipate the impacts of a flood. In this perspective is missing the so called "last mile" that consists in using a hydraulic model that simulates a flood under specific inputs generating specific flooding scenarios that take into account all the possible interactions with the surrounding, also in real time. 
To reach this scope within the HEIMDALL framework are proposed two different hydraulic models to be adopted depending on the phase (prevention, preparedness, etc) and the goal. In fact, two different lines of work were developed:

- A complete 2D hydraulic model (Figure 3)

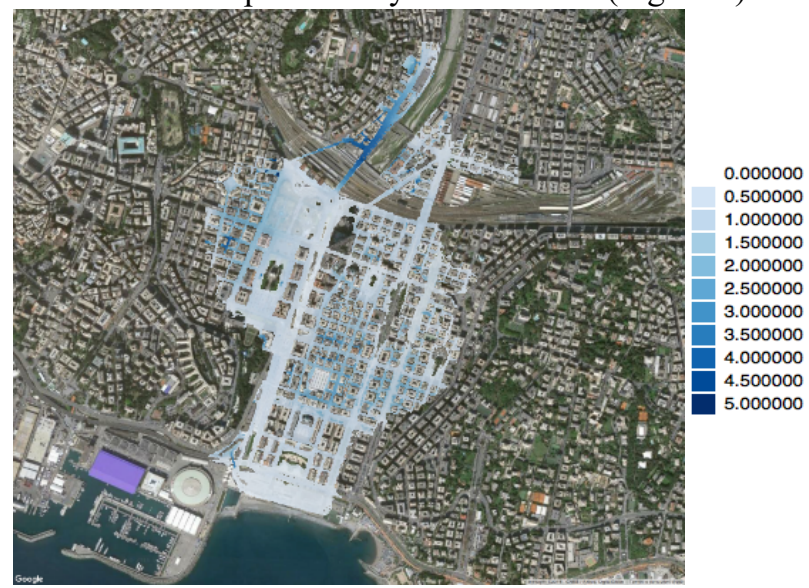

Figure 3: 2D complete hydraulic model of Genoa flood 2014.

- A simplified real-time simulator (Figure 4)

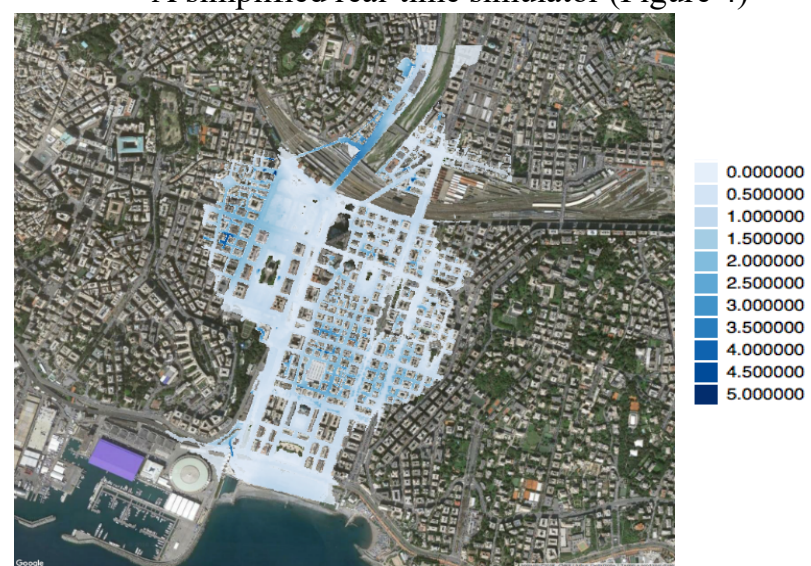

Figure 4: Real-time simulator of Genoa flood 2014.

While the first model consists in a well-known software that allows to generate precise physical simulations using a complete dynamic equation, that needs more time to be set and computational time to be run, the latter model is a simplification of the dynamic equation that requires just few inputs to be quickly run, also obtaining reliable scenarios of flooding.

Moreover, the complete 2D model requires an expert modeller that prepares the requested inputs, prepares all necessary files and runs the model, while the real-time simulator just needs simple inputs to be run. In the HEIMDALL perspective it means that the $2 \mathrm{D}$ complete model needs to be requested and when the results generated are uploaded in the system as GeoTIFF format in order to be visualized, the real-time simulator should be easily managed by an end-user directly clicking on the HEIMDALL user interface.

The usage of a model instead the other is strongly dependent on the goal and civil protection phase. In case we are working in a "peaceful time" the complete model that assures precise scenarios can be used, also of big dimension, that allows organizing and managing a civil protection plan for example. In contrast, if we are working in an emergency situation (caused by weather or by accident) the real-time simulator guarantees a quick but reliable result to take immediate decision.

Both models need inputs to generate the scenarios which are:

- Digital Elevation Model,

- Shape of the river,

- Shape of houses,

- Characteristic of river flood wave (hydrograph peak and duration).

Both models are able to generate outputs with a spatial resolution of a meter and temporal resolution upon request (also of minute) of:

1. Flood extension: maps of flood maximum extension selected from pre-computed database at high spatial resolution,

2. Water depth: maps of water depth in the flooded areas selected from pre-computed database at high spatial resolution,

3. Water velocity: maps of water velocity in the flooded areas selected from pre-computed database at high spatial resolution,

4. Dynamic of flooding: using the previous results in time is possible to evaluate time to reach part of the domain.

Especially the water depth and water velocity outputs should be used as a proxy for the impact assessment module within the HEIMDALL framework.

\subsection{Earth Observation-based flood monitoring}

In the past Earth Observation data have largely demonstrated their potential in disaster management, and are widely used by the decision makers in case of flood events, in particular through operational emergency management mechanisms such as the Copernicus Emergency Management Service (CEMS) Mapping or the International Charter "Space and Major Disasters".

The HEIMDALL platform integrates this kind of data in order to generate and provide reliable and highquality services highlighting the flood extent and its evolution over time, as an input for modelling and risk assessment as well as for supporting emergency response activities. The flood extent products are based on data of several optical and radar satellite sensors, which are automatically or semi-automatically analysed to provide in short timeframe information on the flood affected areas in Europe and worldwide.

Fully automatic flood services based on radar imagery are embedded into HEIMDALL. The TerraSAR$\mathrm{X}$ Flood Service (Martinis et al., 2015) provides local/regional information on demand, with a repeat frequency of 11 days (Figure 5), whereas the Sentinel-1 Flood Service (Twele et al., 2016; Martinis et al., 2018) is 
dedicated to systematic large-scale flood monitoring with a repeat frequency of about 6 days.

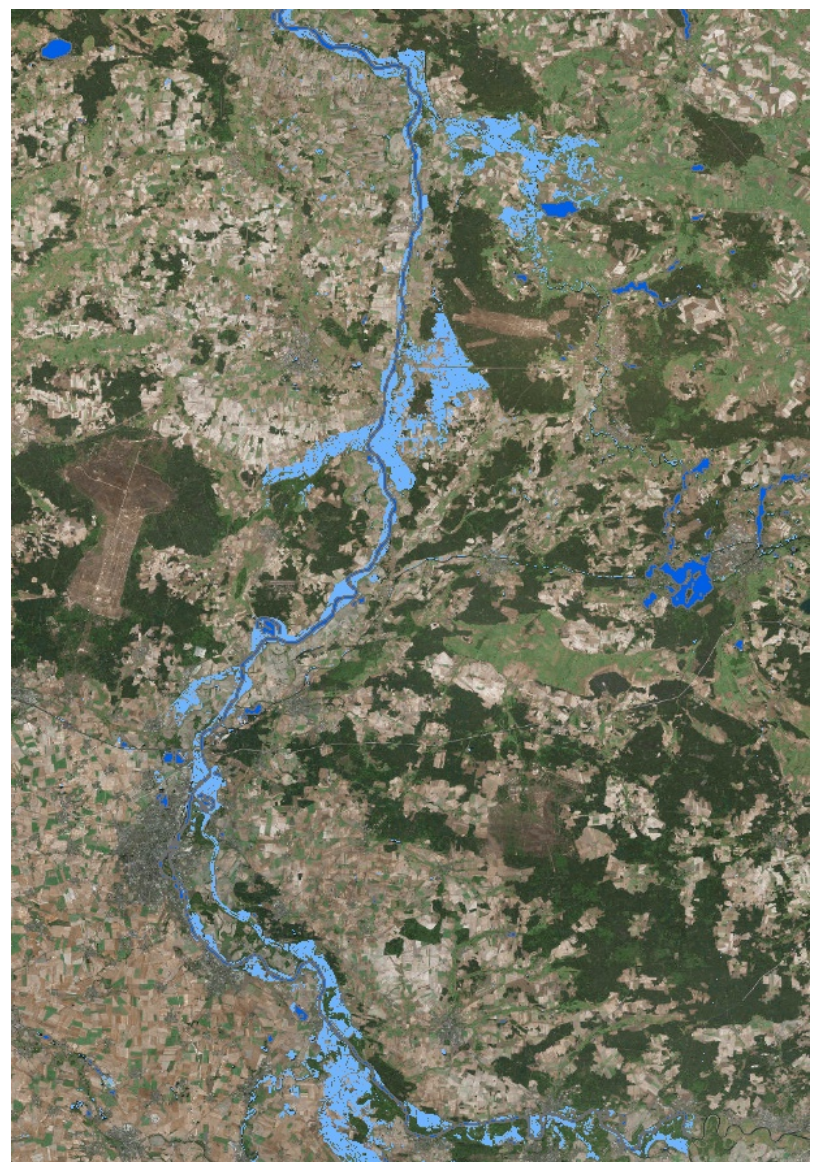

Figure 5. Flood extent derived from TerraSAR-X data (acquisition date 13/06/2013) in Saxony-Anhalt, Germany. Source: German Aerospace Center (DLR).

Radar data are major inputs in floods mapping as they are independent of the weather conditions, whereas optical data are essential for flood analysis in urban areas or after the flood when only flood traces remain visible. Therefore, semi-automatic flood services and processing chains based on optical data are also proposed within HEIMDALL in order to monitor flood extents in urban areas during the response phase. The high resolution optical Sentinel-2 Flood Service provides information on request over large areas, with a repeat frequency similar to Sentinel-1, whereas the very high resolution (VHR) optical processing chain is able to deliver daily information at a very high resolution over targeted areas during cloud free conditions (Figure 6).

The combination of radar and optical multiple satellite sensors increases the observation frequency and the probability to capture the flood extent close to the flood peak.

When a flood event occurs, the decision makers have the possibility to request EO-based products via the HEIMDALL platform by providing the following information: disaster date and time, area of interest, monitoring period if needed, any other helpful information for tasking the satellites (date of flood peak, weather forecast, etc.). The product derived from Earth Observation data is automatically integrated into the
HEIMDALL platform and updated as soon as new data becomes available. The users are notified as soon as a product is available on the platform. The products resulting from the flood services are georeferenced layers highlighting the flood extent over certain time intervals from the beginning of the incident, at medium, high and very high resolutions (up to $30 \mathrm{~cm}$ ).

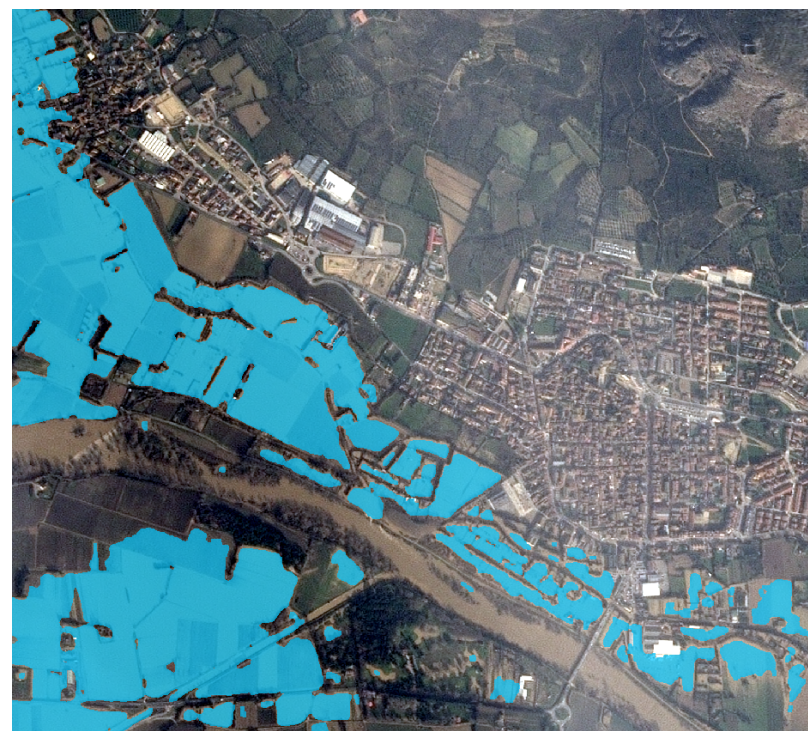

Figure 6: Optical VHR-based flood extent in the area of Torroella de Montgri, Catalonia, Spain, the 24 January 2020. Source: SPOT7 image, (C) CNES 2020, distribution Airbus DS, processing Strasbourg University - ICube SERTIT.

\subsection{Risk and vulnerability}

Risk and vulnerability assessment is one central process needed for effective management of disasters during situation assessment (SA) as well as the planning phase (PF). Risk is assessed through a combination of the probability of a hazardous event and its consequences which result from interaction(s) between natural or manmade hazard(s), the exposed elements and their corresponding vulnerability. Classification of exposed physical elements such as buildings, land use / land cover types, transportation lines, critical infrastructures and the exposed human elements, i.e. people at risk forms the necessary basis for vulnerability and risk assessment.

Both physical and human exposure components are generated using state-of the art techniques incorporating external data sources (see. Figure 1), Volunteer Geographic Information (VGI), remote sensing data and expert knowledge. Highly detailed 3D building models holding information about physical properties such as building height, number of stories as well as their predominant function are retrieved. Building functions available were harmonized using a hierarchical building function taxonomy holding the highest possible level of detail and on the other hand allowing the identification of generic function groups such as residential or nonresidential buildings. During cross-border incidents the integration of datasets with different classification schemes is possible while assuring the functionality of other system components through a consistent data base. 
This allows the definition of flexible top-down disaggregation workflows for the retrieval of high resolution population densities maps and bottom-up modelling approaches to assess structural replacement cost values on building level. Existing national and European land use and land cover products such as e.g. CORINE Land Cover are incorporated. Transportation networks are complementing the physical exposure information, holding information on the location and type of roads and railways present in hazard-prone areas. Several data sources, including national and VGI data sets, are integrated in order to provide the highest possible degree of geometric and semantic information.

The complemented multi-source exposure data set with highly detailed information regarding 3D building models, LULC products, the transportation network, information on critical infrastructures as well as the modelled population density on building level allows comprehensive risk assessment.

Risk is either assessed following a probabilistic approach using simulation and modelling outputs or a deterministic approach based on post-event Earth Observation products implying a probability of 1 . Therefore, the HEIMDALL platform is able to perform risk assessment (during multiple situations) a-priori, analysing simulated flood behaviour and the resulting possible consequences as a measure of risk. The capability of the platform to perform what-if analysis can support decision makers during response and preparedness phase. In addition to a-priori assessment, risk assessment is performed a-posteriori, integrating multi-temporal high-quality products based on EarthObservation data highlighting the flood extent and its evolution over time.

From a general point of view, techniques for the apriori estimation of risk using simulated hazard information are often grouped into three broad categories: qualitative (inventory and knowledge driven), semiquantitative (partially data driven) and quantitative (data driven, deterministic, and probabilistic methods) (see among other Kappes et al., 2012; Coleman and Marks, 1999; Shmidt et al., 2011; Cox et al., 2005; Davidson et al., 2006), though the second technique tends to be a conjunction of the other two categories. The approach here adopted refers to the latter category; more specifically, deterministic/scenario-based analysis is performed. Such kind of analysis is usually applied at local scale due to the process complexity as well as to the required detailed data.

Direct impacts are evaluated through the use of vulnerability functions (also known as damage-state functions); such functions provide a relation in the form of a curve, with an increase in damage for a higher level of hazard intensity. Impact is thus evaluated as in the following:

$$
I \_\boldsymbol{a}^{\wedge} s=f \_\boldsymbol{a}^{\wedge} h\left(w^{\wedge} s\right) * E \_\boldsymbol{a}
$$

where:

$E \_\boldsymbol{a}$ is the value of the asset $\boldsymbol{a}$, typically expressed in monetary form;

$w^{\wedge} S$ is the forcing (measure of the intensity of the hazard) acting in the location where the considered asset is placed in connection to the specific scenario/situation under evaluation;

$f \boldsymbol{a}^{\wedge} h(\cdot)$ is the vulnerability function for asset $\boldsymbol{a}$ in connection to hazard typology $h$; the domain of the function returns percentage values;

I_ $\boldsymbol{a}$ is the direct impact connected to the specific asset (exposed element) $\boldsymbol{a}$ in scenario/situation $s$; it represents the damaged portion of the value of the asset E_a.

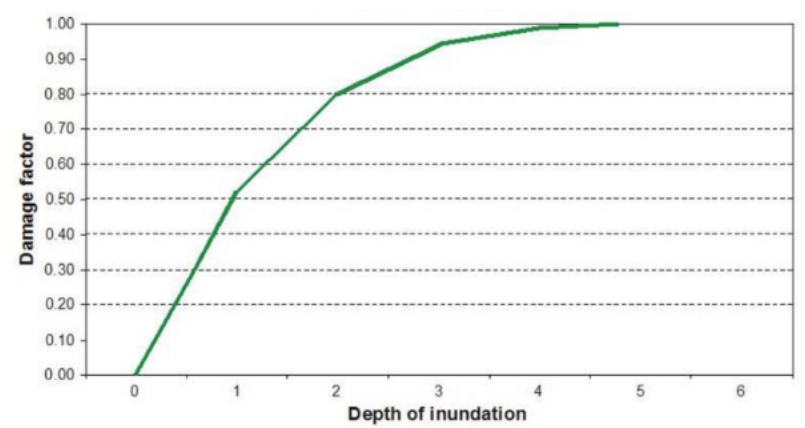

Figure 7: Example of vulnerability function of flood hazard as relation of depth of inundation with respect to damage

Different types of assets will show different levels of damage given the same intensity of hazard; this can be expressed by different shapes of the function $f_{-} \boldsymbol{a}^{\wedge} h(\cdot)$.

Two types of vulnerability functions can be found on literature, historical and synthetic curves. Historical curves are developed from historical loss data from actual events. Synthetic curves instead rely on the analysis of expected damage under certain hypothetical hazard conditions and are used when sufficient past events are not available.

Different functions exist for each type of hazard type of asset, as they may derive from different studies, different geographic peculiarities, and different levels of detail of the analysis to be performed. In this context, asset-level functions are preferred whenever possible. Furthermore, curves expressing the impact as a function of the detailed quantitative description of the hazard are preferred whenever possible, i.e. whenever the simulation models integrated within HEIMDALL provide outputs that are suitable for the use in connection with such type of damage model. Such functions are used for the development of the proper Impact Assessment.

In the specific case of floods, most damage functions are based on the relationship between the type/use of the element at risk and the flood depth. The assumption that is implied is that the dominant effect of the flood is the slow-moving water that is at contact with buildings and objects (Mertz et al. 2010, Kelman and Spence 2004). This is exactly the choice made for the structuring of the main library of vulnerability functions for the complete impact assessment; that uses the building function as key element for the assignment of the functions to the assets, together with the height of the buildings expressed in number of storeys.

For flood impact assessment on buildings, the vulnerability library developed within the RASOR (Koudogbo et al., 2015) project and based on the HAZUS MH Model (Scawthorn et al., 2006, I and II) are 
implemented; such a library has been tested also in (Silvestro et al., 2016) and (Arrighi et al., 2018). The library provides specific functions for different building functions and number of floors. The output is a vector file describing, for each building, the percentage damage; if information on replacement costs is available, also the loss in monetary value is provided.

The combination of Earth Observation based flood extent products derived from satellite imagery acquired during or after a disaster with the exposure information (buildings, road and rail networks, land use land cover, and population) allows to derive physical and human impact assessment products. They can be generated from one observation to assess the impact at a given date and time, or from multi-temporal post-event observations to provide an assessment of the evolution of the impact and the final impact.

The Earth Observation based impact assessment workflows are the result of twenty years of rapid mapping development dedicated to natural disasters management, within international and European frameworks, such as the International Charter "Space and Major Disasters", the Copernicus Emergency Management Service on Rapid Mapping, or the International Working Group on Satellite-based Emergency Mapping (IWG-SEM) which has specified in its Emergency Mapping Guidelines the content and general workflow of impact assessment products, among other things.

The EO based flood impact assessment products define the assets affected or not affected by the flood event, as observed on the satellite imagery. The results can be presented according to the assets properties. The building impact assessment can be displayed according to their use (residential / not residential), their height or number of stories, whereas the population impact assessment provides a number of people (variable scale) per affected building (Figure 8) but other criteria such as the presence of people during day or night can be taken into account.

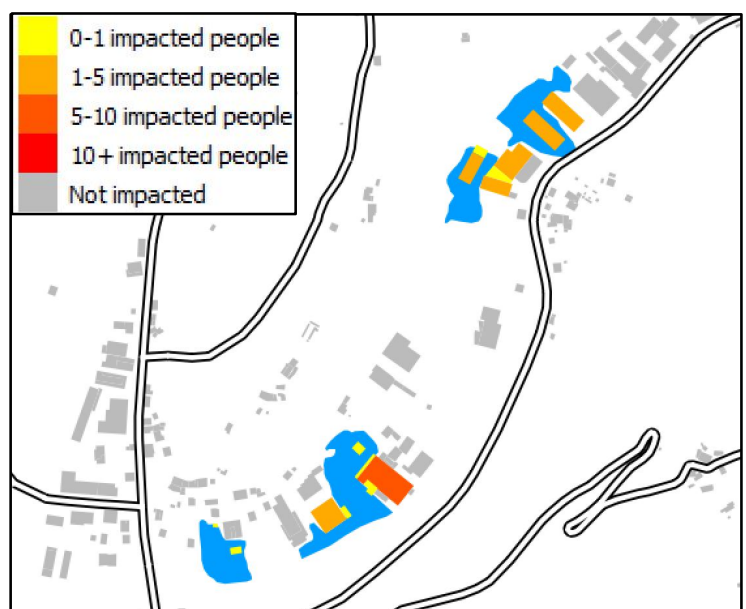

Figure 8: EO based flood impact assessment on population (per building) in Carasco, Italy, as observed the 19 November 2014, one week after the Entella river flood event. Source: PléiadesHR image, (C) CNES 2014, distribution Airbus DS, processing Strasbourg University - ICube SERTIT.
The road network impact assessment highlights the flood affected roads according to their level (primary, secondary, tertiary, etc.), and the land use / land cover (LULC) impact assessment presents the flood impact according to the LULC categories at different levels (e.g. level 1 corresponds to the main categories, such as artificial, agricultural, forest, etc.).

\subsection{Situation assessment}

In the SA phase of decision making, maintaining a sound and complete situational awareness of an emerging or occurring disaster is an essential prerequisite for effective decision making to minimize the consequences of a disaster. The situational picture consisting of information generated by the HEIMDALL modules and by the users can be saved at different points in time in scenario snapshots enabling tracking and reproduction of the situation evolution and changes in the environment such as changing weather conditions or hazard behaviour. Case studies on the assessment of emergency plans for floods revealed a need for further elaboration of supporting relevant information in the process of emergency planning (Lumbroso et al., 2010). The major issues identified included the importance of having information on demographic numbers, on the location of vulnerable infrastructure and people and of being able to connect the different automated systems used by the different parties involved in emergency planning. The HEIMDALL module ISAS combines flood (or fire/landslide) simulations and impact assessment results with strategical geographical objects which can be predefined and customized by the end users. As a result, the module provides an impact summary for a specified area of interest with total numbers of affected buildings and roads, physical damage, people at risk (day/night) and economic damage. In addition, users are provided with a list of affected geographical objects of interest (GOIs) such as buildings and roads customizable according to organizational strategies and constraints, e.g. mark a GOI as significant infrastructure. The impact summary is displayed in descriptive form in the user interface and can be exported over its REST API. The lists of affected GOIs can be filtered and downloaded in a tabular format (i.e. CSV). For example, end users may request a list of affected schools and hospitals in an area they know is prone to flooding.

The HEIMDALL situation reporting service (SITREP) transforms scenarios including all relevant, related information into standardized EDXL-SitRep messages. The goal is to create means for sharing a common operational picture in a standards-based way by fostering interoperability between systems. The idea is that a system client in a specific environment (e.g. mobile app on a field responder's phone) is able to request situational awareness information from a HEIMDALL unit in a different location, e.g. in a control room in a different country. If that client includes a tool to interpret the message protocol and format, it is able to present information included in the message in any desired format, such as text, PDF or map layer. 
The EDXL "Community Extension" (short "extension") mechanism allows for supplemental inclusion of community-defined sets of name/value pairs where needed. For example, if the end user sends a SitRep message with an image about a simulated flood extent, he/she might want to include some specific flood simulation data, like the mean anticipated water height and velocity. We use extensions in order to include simulation results data, weather forecasts (in addition to current weather conditions) and sub-hazard types defined in HEIMDALL. The extension concept improves information sharing between local authorities and developing standards for the emergency management community. What works well for communities can be incorporated formally into future standards.

The impact summary functionality can be used together with simulation and impact assessment tools to analyse possible future scenarios. These scenario options start with different foreseen hazard evolutions and potential measures of prevention and control as an input in order to explore the consequences of impacts and measures to be performed in these options. The HEIMDALL system can assist decision makers to generate alternative scenarios (using scenario management functions) and assess their potential evolution and consequences using simulation, impact assessment and ISAS functions. Decision makers such as incident commanders in the field can display and explore these alternative scenarios in the user interface or browse through them when they are presented in the form of a situation report (e.g. generated by SITREP). Based on such a situation report which contains a comparison of the top best-fitting planning scenarios, each with relevant information on conditions and consequences, their knowledge of the context and their experience they are able to take an informed decision on the best-fitting scenario option and the appropriate working strategy.

\subsection{Decision support}

In the PF phase, information on options and contingencies is needed: if the flood situation escalates, what is required? If a major transportation route is at risk, where in the vicinity are alternative roads which are not affected? If casualties are recovered, is there somewhere to take them?

It strongly depends on the processes to be supported, their legal and organizational context, their degree of structuredness and the existence of best practices and SOPs that may be automated whether decision support systems (DSS) leave the generation of situational awareness to the user (less automatic) or whether they provide fully automatic decision options among which the user has just to choose. While the latter has proven to be helpful in numerous operational tsunami early warning situations (Steinmetz et al., 2010), longterm response planning activities identified so far in the HEIMDALL context (Friedemann et al., 2018) require a much more data-driven approach without decision automation. In the field of data driven DSS the key is having easy and rapid access to a large amount of accurate, well-organized multidimensional data (Power, 2008). Based on this data users are able to perform situation and risk analysis, assessment and strategic planning more efficiently. This applies particularly for wildfire and flood hazards which materialize over days before they get imminent thus require a high degree of user expertise in their management. As a result, the focus of computerized decision support has been shifted from automated system actions (e.g. decision proposals for routes of actions, tasking of models and simulations) to the preparation and provision of relevant decision support (DES) information.

To this end, scenario matching results constitute relevant DES information. Furthermore, DES information on potentially safe (potentially not affected for a given time) task-oriented infrastructure, customized towards the addressing organization, is generated based on a fusion of expert, scenario, simulation, and impact assessment information for real and fictional scenarios. Examples are safe potential shelters, safe roads and bridges and possible target areas for evacuation.

\section{Benefits of HEIMDALL from the point of view of the first responders}

The complexity of natural disasters is evident. In this sense, HEIMDALL delivers great benefits for both preparedness and response phases, and furthermore within the three different levels of response: strategic, tactical and operational. Besides that, each of the groups acting in an emergency can train their personnel in their specific functions and operations. Training mode is an interactive and illustrative way to show different scenarios with different risks, complexities, or characteristics at the same time that they can be taught to use the different functionalities, sources of information or even communicative techniques necessary in a real emergency.

HEIMDALL services and tools are designed, to cover the various needs of emergency management actors. This is achieved by gathering user expectations and requirements in a collaborative design methodology.

In the preparedness phase, HEIMDALL allows users to gather and analyse specific hazardous conditions (weather data, wildfire risk indexes, etc.) in anticipation of a potential incident as well as consult former disaster scenarios with similar characteristics that have been previously stored and shared in the scenario repository. These scenarios are based on user experience and allow them to associate lessons learnt from, decisions taken, and response plans, among other information, of former incidents. During the response phase, HEIMDALL improves situation awareness and facilitates enhanced risk assessment of the ongoing incident in real time. For instance, by of the integration of modelling and simulation tools into the system, combined with data fusion techniques, HEIMDALL users can follow the evolution of the advancing hazard as it unfolds or continuously assess the vulnerability of exposed population. As a result, the incident commander can evaluate the resources available, the objectives set, record 
the actions taken, monitor operations and the situation at a strategic level and cooperate with other agencies through agreed coordination protocols and previously established channels.

At an operational and tactical level, an incident commander will have real-time information about the situation, its evolution and possible impacts on assets or people. This facilitates decision making and the evaluation of the adopted strategy.

By using HEIMDALL the first responders in the field have real-time information on the evolution of the risk, thereby ensuring the safety and efficiency of actions required during the emergency. In addition, all the information that is entered in the platform will enrich the repository of scenarios with information that can be retrieved in future incidents that match similar characteristics, thus contributing to make future actions more effective. Another important emergency management aspect to consider is the communication strategy, as HEIMDALL provides reliable information to share with politicians, media or the general public.

In conclusion, natural destructive phenomena cannot be avoided but the consequences and the negative impacts can be mitigated. By means of a novel technological approach, HEIMDALL aims to facilitate cooperation and coordination among agencies, assisting them with risk information to prepare for and respond to emergencies caused by natural disasters. Along these lines, not only preparation and response are improved, but also the capacity for recovery and adaptation.

\section{Summary and Perspectives}

\footnotetext{
HEIMDALL's Multi-Hazard Cooperative Management Tool for Data Exchange, Response Planning and Scenario Building is aimed at facilitating all phases of disaster management from prevention/mitigation, through crisis management to recovery. Through extensive work with engaged users, the HEIMDALL platform is beginning to offer userfriendly modules covering a whole array of functions including: Data Source Access, Scenario Management, Modelling and Simulation, Risk and Vulnerability, Impact Assessment, Situation Assessment, Decision Support, Data Sharing and Communication. Under this frame, HEIMDALL partners have outlined the capabilities of the system within the context of Flood management, a topic of the utmost importance.

First versions of the HEIMDALL platform have been presented in several workshops and tested by various end-users. The final release will follow by the end of the project with the objective of publishing a pre-operational platform at the end of 2020 which could be used by HEIMDALL's end-users, before extending it to additional users. Moreover, the system should be extended in the near future to cover other natural and human-made hazard types. Further information will follow: http://heimdall-h2020.eu/.
}

\section{Acknowledgement}

This project has received funding from the European Union's Horizon 2020 research and innovation programme under grant agreement No 740689.

\section{References}

1. Alexander, D. (2009). Principles of emergency planning: standardisation, integration and sustainability. In: Building safer communities: Risk governance, spatial planning and responses to natural hazards, edited by: Fra Palco, U., NATO science for peace and security series, IOS Press, Amsterdam, pp. 162-174.

2. Arrighi, C., L. Rossi, E. Trasforini, R. Rudari, L. Ferraris, M. Brugioni, S. Franceschini and F. Castelli (2018). Quantification of Flood risk mitigation benefits: A building-scale damage assessment through the RASOR platform. Journal of environmental management, vol. 207, pp. 92-104.

3. Behrens, J., A. Androsov, A.Y. Babeyko, S. Harig, F. Klaschka and L. Mentrup (2010). A new multisensor approach to simulation assisted tsunami early warning. Natural Hazards and Earth System Sciences 10, pp. 1085-1100, doi:10.5194/nhess-101085-2010.

4. Cohen-Hatton, S.R., P.C. Butler and R.C. Honey (2015). An Investigation of Operational Decision Making in Situ Incident Command in the UK Fire and Rescue Service. J. Hum Factors, 57, pp. 793804, doi:10.1177/0018720815578266/.

5. Coleman, M. and H. Marks (1999). Qualitative and quantitative risk assessment. Food Control, vol. 10, pp. 289-297.

6. Copernicus Emergency Management Service Mapping. https://emergency.copernicus.eu/mapping/

7. Copernicus Emergency Management Service European Forest Fire Information System (EFFIS). https://effis.jrc.ec.europa.eu/.

8. Cox Jr, L.A., D. Babayev and W. Huber (2005). Some limitations of qualitative risk rating systems. Risk Analysis: An International Journal, vol. 25, pp. 651-662.

9. Davidson, V.J., J. Ryks and A. Fazil (2006). Fuzzy risk assessment tool for microbial hazards in food systems. Fuzzy Sets and Systems, vol. 157, pp. 12011210.

10. De Brito, M.M. and M. Evers (2016). Multi-criteria decision-making for flood risk management: a survey of the current state of the art. Natural Hazards and Earth System Sciences, 16(4), pp. 1019-1033.

11. European Commission - DG Environment (2013). Technical Support in Relation to the Implementation of the Floods Directive (2007/60/EC). A User Guide to the Floods Reporting Schemas. http://cdr.eionet.europa.eu/help/Floods/Floods 603 2016/resources/User\%20Guide $\% 20$ to $\% 20$ the $\% 20 \mathrm{Fl}$ oods\%20schema\%20v6.0.pdf

12. Friedemann, M., B. Barth, J. Vendrell, M. Muehlbauer and T. Riedlinger (2018). Conceptual 
scenario model for collaborative disaster response planning. Bungartz, H.-J., Kranzlmueller, D., Weinberg, V., Weismueller, J., Wohlgemuth, $V$. (Eds.): Environmental Informatics: Techniques and Trends, Adjunct Proceedings of the 32nd edition of the EnviroInfo, pp. 119-125.

13. Gabellani, S., F. Giannoni, A. Parodi, R. Rudari, A. C. Taramasso and G. Roth (2005). Applicability of a forecasting chain in a different morphological environment in Italy. Advances in Geosciences, 2, pp. 131-134.

14. Goodridge, W.S. (2016). Sensitivity analysis using simple additive weighting method. International Journal of Intelligent Systems and Applications, 8(5), 27.

15. Hally, A., O. Caumont, L. Garrote, E. Richard, A. Weerts, F. Delogu and M. Ivković (2015). Hydrometeorological multi-model ensemble simulations of the 4 November 2011 flash flood event in Genoa, Italy, in the framework of the DRIHM project. Natural Hazards and Earth System Sciences, 15(3), pp. 537-555.

16. Han, J., M. Kamber and J. Pei (2006). Data Mining: Concepts and Techniques. Elsevier Science \& Technology, Burlington, United States.

17. HEIMDALL (2017), HEIMDALL - Multi-Hazard Cooperative Management Tool for Data Exchange, Response Planning and Scenario Building. http://cordis.europa.eu/project/rcn/210221 de.html, http://heimdall-h2020.eu/.

18. HEIMDALL (2018), Deliverable D2.11: Service Concept Specification. http://heimdall-h2020.eu/wpcontent/uploads/2018/05/HEIMDALL_D2.11.DLR_. v1.O.F.pdf: 19.

19. HEIMDALL (2018), Deliverable D2.12: HEIMDALL System Architecture. http://heimdallh2020.eu/wpcontent/uploads/2018/05/HEIMDALL_D2.12.DLR_. v1.0.F.pdf: 10.

20. House, A., N. Power and L. Alison (2014). A systematic review of the potential hurdles of interoperability to the emergency services in major incidents: Recommendations for solutions and alternatives. Cognition, Technology \& Work 16, 3 (August 2014), pp. 319-335.

21. International Charter "Space and Major Disasters". https://disasterscharter.org/

22. International Working Group on Satellite-based Emergency Mapping (IWG-SEM). http://www.unspider.org/network/iwg-sem

23. Kappes, M.S., M. Keiler, K. von Elverfeldt and T. Glade (2012). Challenges of analyzing multi-hazard risk: a review. Natural hazards, vol. 64, pp. 19251958.

24. Karney, C.F.F. (2013). Algorithms for geodesics. Journal of Geodesy 87, pp. 43-55, doi:10.1007/s00190-012-0578-z.

25. Kelman I. and R. Spence (2004). An overview of flood actions on buildings. Engineering Geology, vol. 73, pp. 297-309.
26. Koudogbo, F.N., R. Rudari, A. Eddy, E. Trasforini, L. Rossi, H. Yésou, J. Beckers, F. Dell'acqua, M. Huber, A. Roth, S. Salvi, A. Ganas (2015). EO data for rapid risk analysis with the RASOR platform. IEEE International Geoscience and Remote Sensing Symposium (IGARSS).

27. Lumbroso, D.M., M. Di Mauro, A.F. Tagg, F. Vinet and K. Stone (2012). FIM FRAME: a method for assessing and improving emergency plans for floods. Natural Hazards and Earth System Sciences., 12, pp. 1731-1746, doi:10.5194/nhess-12-1731-2012.

28. Martinis, S., A. Twele and J. Kersten (2015): A fully automated TerraSAR-X based flood service. ISPRS Journal of Photogrammetry and Remote Sensing, 104, pp. 203-212.

29. Martinis, S., S. Plank and K. Cwik (2018). The use of Sentinel-1 time-series data to improve flood monitoring in arid areas. Remote Sensing, 10, 583.

30. Martinis, S., S. Plank and K. Cwik (2018). The use of Sentinel-1 time-series data to improve flood monitoring in arid areas. Remote Sensing, 10, 583.

31. Merz, B., H. Kreibich, R. Schwarze and A. Thieken (2010). Review article "Assessment of economic flood damage". Natural Hazards and Earth System Sciences, vol. 10, 1697.

32. Molini, L., A. Parodi and F. Siccardi (2009). Dealing with uncertainty: an analysis of the severe weather events over Italy in 2006. Natural Hazards and Earth System Sciences, 9(6), pp. 1775-1786.

33. Poletti, M.L., A. Parodi and B. Turato (2017). Severe hydrometeorological events in Liguria region: calibration and validation of a meteorological indices based forecasting operational tool. Meteorological Applications, 24(4), pp. 560-570.

34. Power, D.J. (2008). Understanding Data-Driven Decision Support Systems. Information Systems Management, $\quad 25: 2, \quad 149-154$, doi:10.1080/10580530801941124.

35. Scawthorn, C., N. Blais, H. Seligson, E. Tate, E. Mifflin, W. Thomas, J. Murphy and C. Jones (2006). HAZUS-MH Flood Loss Estimation Methodology. I: Overview and Flood Hazard Characterization, vol. 7, pp. 60-71.

36. Scawthorn, C., P. Flores, N. Blais, H. Seligson, E. Tate, S. Chang, E. Mifflin, W. Thomas, J. Murphy, C. Jones and M. Lawrence (2006). HAZUS-MH Flood Loss Estimation Methodology. II. Damage and Loss Assessment, vol. 7, pp. 72-81.

37. Schmidt, J., I. Matcham, S. Reese, A. King, R. Bell, R. Henderson, G. Smart, J. Cousins, W. Smith and D. Heron (2011). Quantitative multi-risk analysis for natural hazards: a framework for multi-risk modelling. Natural Hazards, vol. 58, pp. 1169-1192.

38. Siccardi, F., G. Boni, L. Ferraris and R. Rudari (2005). A hydrometeorological approach for probabilistic flood forecast. Journal of Geophysical Research: Atmospheres, 110(D5).

39. Silvestro, F., N. Rebora and L. Ferraris (2011). Quantitative flood forecasting on small-and mediumsized basins: a probabilistic approach for operational purposes. Journal of Hydrometeorology, 12(6), pp. 1432-1446. 
40. Silvestro, F., N. Rebora, L. Rossi, D. Dolia, S. Gabellani, F. Pignone, E. Trasforini, R. Rudari, S.D. Angeli, and C. Masciulli (2016). What if the 25 October 2011 event that struck Cinque Terre (Liguria) had happened in Genoa, Italy? Flooding scenarios, hazard mapping and damage estimation. Natural Hazards and Earth System Sciences, vol. 16, pp. 1737-1753.

41. Steinmetz, T., U. Raape, S. Teßmann, C. Strobl, M. Friedemann, T. Kukofka, T. Riedlinger, E. Mikusch, S. Dech (2010). Tsunami early warning and decision support. Natural Hazards and Earth System Science 10, pp. 1839-1850, doi:10.5194/nhess-10-1839-2010

42. Twele, A., W. Cao, S. Plank and S. Martinis (2016). Sentinel-1 based flood mapping: a fully automated processing chain. International Journal of Remote Sensing, 37 (13), pp. 2990-3004.

43. UNISDR (2015). The human cost of weather-related disaster. Analysis report. 